\title{
EXPERIENCE-DEPENDENT CHANGES IN CEREBRAL FUNCTIONAL CONNECTIVITY DURING HUMAN RAPID EYE MOVEMENT SLEEP
}

Laureys $S^{(1)(2)}$, Peigneux $P^{(1)(2)(3)}$, Phillips $C^{(1)}$, Fuchs $S^{(2)}$, Degueldre $C^{(1)}$, Aerts $J^{(1)}$, Del fiore $\mathrm{G}^{(1)}$, Petiau $\mathrm{C}^{(1)}$, Luxen $\mathrm{A}^{(1)}$, Van $\operatorname{der} \operatorname{linden} \mathrm{M}^{(3)}$, Cleeremans $\mathrm{A}^{(4)}$, Smit $\mathrm{C}^{(5)}$, Maquet $\mathrm{P}^{(1)(2)(6)}$

(1)Cyclotron Research Center, University of Liège, Liège, Belgium

(2)Department of Neurology, CHU Sart Tilman, 4000 Liège, Belgium

(3)Department of Neuropsychology, University of Liège, Liège, Belgium

(4)Cognitive Science Research Unit, Universite Libre de Bruxelles, Brussels, Belgium

(5)Department of Psychology, Trent University, Peterborough, QC, Canada

(6)Wellcome Department of Cognitive Neurology, University College London, London, UK

\begin{abstract}
One function of sleep is hypothesized to be the reprocessing and consolidation of memory traces (Smith, 1995; Gais et al., 2000; McGaugh, 2000; Stickgold et al., 2000). At the cellular level, neuronal reactivations during post-training sleep in animals have been observed in hippocampal (Wilson and McNaughton, 1994) and cortical (Amzica et al., 1997) neuronal populations. At the systems level, using positron emission tomography, we have recently shown that some brain areas reactivated during rapid-eye-movement sleep in human subjects previously trained on an implicit learning task (a serial reaction time task) (Maquet et al., 2000). These cortical reactivations, located in the left premotor area and bilateral cuneus, were thought to reflect the reprocessing — possibly the consolidation - of memory traces during post-training rapid-eye-movement sleep. Here, the experience-dependent functional connectivity of these brain regions is examined. It is shown that the left premotor cortex is functionally more correlated with the left posterior parietal cortex and bilateral pre-supple-mentary motor area during rapid-eye-movement sleep of subjects previously trained to the reaction time task compared to rapid-eye-movement sleep of untrained subjects. The increase in functional connectivity during post-training rapid-eye-movement sleep suggests that the brain areas reactivated during posttraining rapid-eye-movement sleep participate in the optimization of the network that subtends subject's visuo-motor response. The optimization of this visuo-motor network during sleep could explain the gain in performance observed during the following day.
\end{abstract}

Key words: memory, brain plasticity, psychophysiological interaction analysis, functional neuroimaging, positron emission tomography.

\section{Abbreviations}

MRI, magnetic resonance imaging; PET, positron emission tomography; rCBF, regional cerebral blood flow: REM, rapid-eye-movement sleep; SMA, supplementary motor area.

We found that during rapid-eye-movement (REM) sleep of subjects previously trained to a serial reaction time task, compared to subjects without pre-sleep practice, the left dorsal premotor cortex (identified in our previous study; Maquet et al, 2000) experiences an increased functional connectivity with the left posterior parietal cortex (Brodmann area 7) and bilateral pre-supplemen-tary motor area (pre-SMA; Brodmann area 6) (see Fig. 1 and Table 1). Both posterior parietal cortex (Wise et al., 1997) and pre-SMA (Pandya and Vignolo, 1971; Luppino et al, 1993) are known to be structurally connected to the premotor cortex. The left cuneus showed a difference in modulation with/from the right dorsal pre-motor (stereotaxic coordinates 42, 22, 52; Talairach and Tournoux, 1988) and frontopolar regions (coordinates 26, 64, 18). Because neither area was included in our a priori anatomical hypotheses (see Experimental procedures), these results are reported for completeness but will not be discussed further. The right cuneus showed no significant differences in functional connectivity with other brain regions. Brain function relies on both functional segregation and integration (Friston, 1997). Segregation 
indicates that specific brain areas are specialized in the treatment of certain types of information, while integration emphasizes that cerebral functions emerge from the interactions between various brain areas (e.g. changes in functional connectivity).
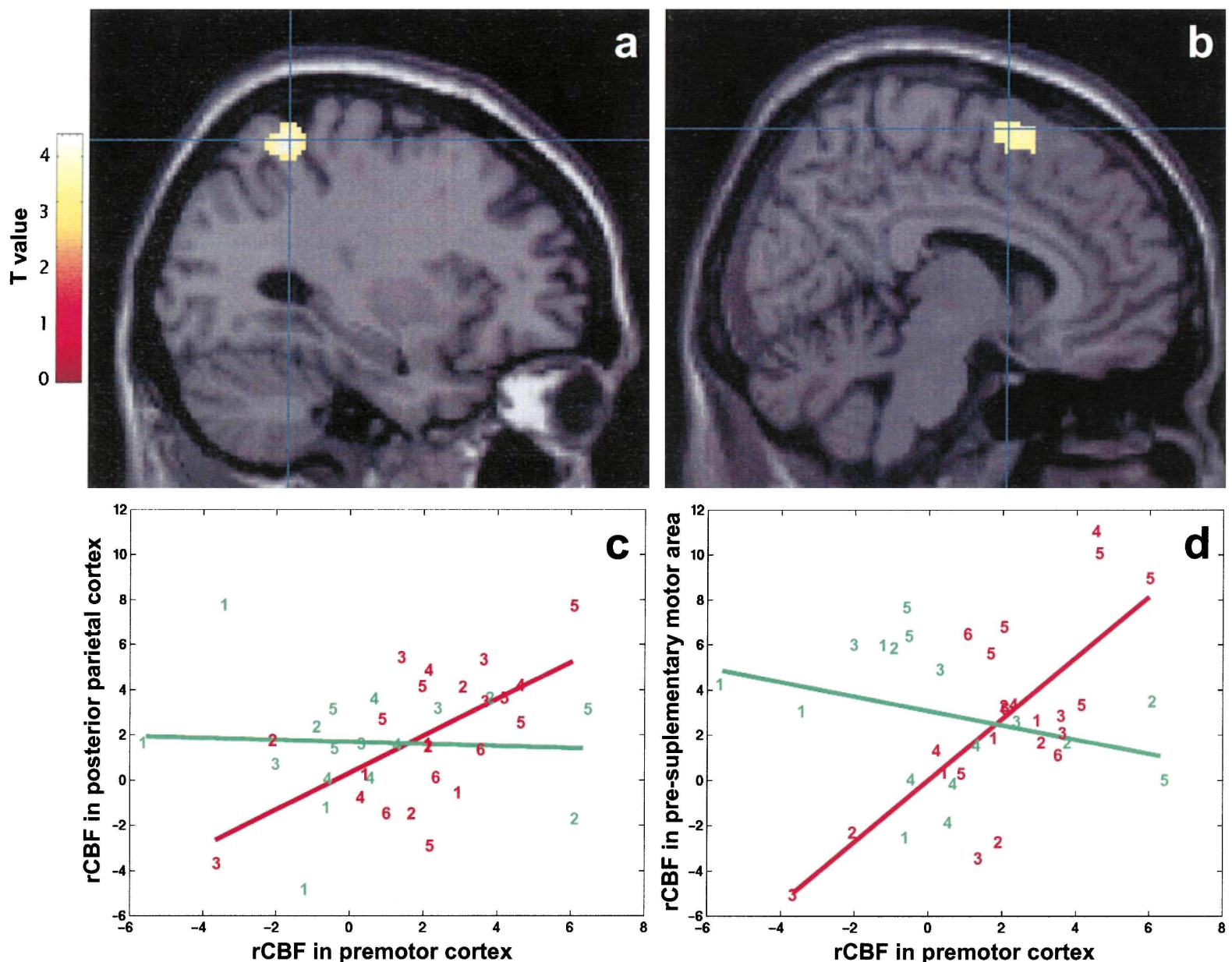

Fig. 1. Upper panels: (a) Posterior parietal and (b) pre-SMAs that showed a significant $(P<0.001)$ increase in functional connectivity with the premotor cortex during REM sleep in trained versus untrained subjects, projected on a sagittal section of a normalized brain MRI. Lower panels: Plot of the regression of centered $\mathrm{rCBF}$ in left premotor cortex (stereotaxic coordinates $-24,4,64)$ and (c) the posterior parietal cortex (coordinates $-30,-48,60)$ and (d) the pre-SMA (coordinates 2, 6, 64) during post-training REM sleep (red) and REM sleep from untrained subjects (green). Numbers represent the numbers of the individual subjects from each group

Functional connectivity, or the correlation between remote and neurophysiological events does not imply any directionality or causal inference. However, in a psychophysiological interaction (Friston et al., 1997), responses in a target area can be explained by the interaction between a psychological factor and activity in a source area. This implies directionality in the sense that activity in the source area could not, necessarily, be explained by an interaction between the psychological factor and activity in the target. However, within any one level of the psychological factor the coupling inferred is not directional and non-causal. A psychophysiological interaction is essentially testing for a difference in the regression slope of activity in the target area on activity in the source area under different levels of a psychological factor. These regression slopes can be thought of as a measure of coupling in the sense that they reflect the change in the target for a unit change in the source. Therefore, a psychophysiological interaction implies that this measure of coupling is influenced or modulated by the psychological variable. In this case the psychological factor corresponds to pre-sleep training and our hypothesis is that this will increase the coupling among important components of the visuomotor system.

Our results show enhanced functional relationships between the dorsal premotor cortex and the posterior parietal cortex (anterior part of the intraparietal sulcus) and pre-SMA during post-training REM sleep compared to REM sleep in untrained subjects. The main point here is that at least some of 
the cerebral areas reactivated during post-training sleep do not act in isolation. In contrast, their reactivation reflects the recruitment of a large-scale neuronal network recently challenged by a new environmental situation. The posterior parietal cortex and pre-SMA are described in the monkey as regions that are part of the multiple parallel parieto-frontal circuits devoted to sensorimotor transformations (Houk and Wise, 1995). They are hypothesized to be similarly involved in the transformation of sensory information into action in humans (Rizzolatti and Arbib, 1998). The human posterior parietal cortex contains several distinct cortical areas, strongly interconnected via corticocortical projections. They combine modalities and participate in the elaboration of spatial representation (Andersen, 1997) and body reference (Wolpert et al., 1998), play a pivotal role in transforming visual inputs into motor plan (Rizzolatti et al, 1998) and support on-line visual control for fast corrective movements (Pisella et al., 2000).

Table 1. Stereotaxic coordinates of areas that showed an increase in functional connectivity with left premotor cortex during REM sleep in subjects previously trained to a serial reaction time task versus untrained subjects.

$\begin{array}{lllllll}\begin{array}{l}\text { Identified region } \\ \text { Left posterior parietal }\end{array} & \text { Brodmann area } & X & y & z & \text { Z value } & \begin{array}{l}\text { Small volume corrected } P \\ \text { value }\end{array} \\ \begin{array}{l}\text { cortex } \\ \text { Pre-SMA }\end{array} & 7 & -30 & -48 & 60 & 3.71 & 0.008 \\ & 6 & 2 & 6 & 64 & 3.45 & 0.014\end{array}$

Pre-SMA is known to contribute to sequence learning (Boecker et al, 1998) and recent evidence suggests that pre-SMA would be more specifically involved in perceptual-motor associations (Sakai et al, 1999; Kurata et al, 2000). Both the posterior parietal cortex and pre-SMA were previously shown to be involved in the visuo-motor processes elicited by practice of the serial reaction time task (Maquet et al, 2000) in which the motor response is determined by the visually displayed location of the stimulus (local maxima of posterior parietal and pre-SMA are less than $10 \mathrm{~mm}$ from those identified when subjects learned the task during waking).

The increased functional connectivity during post-training REM sleep between areas involved in visuomotor transformations and motor behavior supports the hypothesis of the refinement of a neural network, probably related to the subjects' learning of the serial reaction time task. The present results provide further support for the hypothesis that recent memory traces, embodied in the networks challenged by new environmental conditions are processed during subsequent sleep. It is important to stress that they do not exclude the contribution of slow-wave sleep to memory formation during sleep in humans (Gais et al., 2000; Stickgold et al., 2000). Whether and how this processing actually serves to consolidate the recent memory traces remains to be further elucidated.

\section{Experimental procedures}

\section{Experimental protocol}

The study was approved by the Ethics Committee of the Faculty of Medicine of the University of Liege. All experiments were conducted in accordance with the Declaration of Helsinki and written informed consent was obtained from all subjects. They were young (mean $22.9 \pm 3.5$ years), healthy, right-handed males. The experimental protocol has been more extensively described elsewhere (Maquet et al., 2000). In summary, trained subjects $(n=6)$ learned a serial reaction time task (see below), between 16:00 and 20:00, and, on the following day, between 16:00 and 18:00. During the intervening night, 12 positron emission tomography (PET) scans were performed both during waking and during sleep. Sleep scans were performed when polysomnography showed steady characteristic sleep patterns. The untrained subjects $(n=5)$ followed exactly the same protocol, except that they were asked to remain in the laboratory and to have no intensive or continuous activity between 16:00 and 20:00, prior to the night of the PET study.

\section{Serial reaction time task}

A detailed description of the serial reaction time task can be found elsewhere (Cleeremans and McClelland, 1991; Peigneux et al., 2000). Participants faced a computer screen where six permanent position markers were displayed. A keyboard with six spatially compatible response keys was within reach of the right hand. Subjects were instructed to react as fast and as accurately as possible to the 
appearance of a stimulus below one of the markers by pressing on the spatially corresponding key. The next stimulus was then displayed after a fixed interval of $200 \mathrm{~ms}$, until completion of a block of 205 trials. Forty-eight reaction time blocks were practiced before the night of the PET study, 24 blocks were practiced the day after. Block mean reaction time significantly improved on the post-sleep session as compared to pre-sleep performance (Maquet et al., 2000) suggesting that subjects in the trained group refined their task-dependent visuo-motor capabilities overnight.

\section{Polysomnography acquisition and analysis}

Polysomnography was recorded with a Synamp (Neuroscan. NeuroSoft, Sterling, VA, USA), at 500 $\mathrm{Hz}$, with a bandwidth 0.15 to $100 \mathrm{~Hz}$ and $\mathrm{Al}$ as a reference. For the third night, 28 scalp channels were placed according to the 10-20 system: Vertical and horizontal electrooculograms, chin electromyogram and electrocardiogram were recorded on bipolar montages. Polygraphic recordings were scored using standard criteria (Rechtschaffen and Kales, 1968).

\section{PET acquisition and analysis}

PET data were acquired on a Siemens CTI 951 R 16/31 scanner in 3D mode. The subject's head was stabilized by a thermoplastic facemask (Truscan imaging, MA, USA) and a venous catheter was secured in a left antebrachial vein. A transmission scan was acquired for attenuation correction.

Regional cerebral blood flow (rCBF) was estimated using the $\mathrm{H}_{2}{ }^{15} \mathrm{O}$ infusion method (Maquet et al., 2000). Tl-weighted magnetic resonance imaging (MRI) (0.96 x $0.96 \times 1.50 \mathrm{~mm}$ voxel size) was performed on a 1.5-T Magnetom scanner (Siemens, Erlangen, Germany). PET data were realigned, coregistered, normalized, smoothed (16 mm full width at half maximum) and analyzed using statistical parametric mapping (SPM99; Welcome Department of Cognitive Neurology, Institute of Neurology, London, UK). The present analysis is based on the REM sleep scans obtained in trained (23 scans) and untrained subjects (17 scans) (i.e. three to six REM scans per subject). Proportional scaling adjusted rCBF for changes in global flow. The effect of the covariates of interest was estimated according to the general linear model at each voxel. The covariates consisted of the group effect (trained versus untrained) and the $\mathrm{rCBF}$ of the three reference regions previously found to reactivate during REM sleep in trained subjects as compared to naive subjects (Maquet et al., 2000): the left dorsal premotor cortex (coordinates $-24,4,64$ ). the left cuneus (coordinates -18, -80, 24) and the right cuneus (coordinates 22 , $-70,16)$. The activity in the reference regions was modeled separately for the two groups, allowing us to test for an interaction in terms of the difference in the regression slopes between the two groups. This analysis identified the brain areas that were functionally more related to each of the reference areas in trained than in untrained subjects during REM sleep. These changes in coupling, however, could reflect either a within subject co-activation of the coupled regions over the REM scans from each subject, or a systematic covariation over subjects depending upon the degree of experience-dependent plasticity, or both. In the present study it is difficult to partition the source of covariation given the small numbers of replications from within each subject.

The analysis of functional integration through the assessment of psychophysiological interactions (Friston et al., 1997) must rely on the available knowledge about the structural connections between the reference cerebral areas and the rest of the brain. Hence, only the brain areas known to be neuroanatomically connected to the cuneus and the premotor cortex were considered. In non-human primates, the premotor cortex is connected (most often reciprocally) with the posterior and medial parietal cortices (Wise et al., 1997), the dorsolateral prefrontal cortex (Lu et al., 1994), the SMA and pre-SMA (Pandya and Vignolo, 1971; Luppino et al., 1993) and the precentral gyrus (Pandya and Vignolo, 1971). Subcortical connections of the premotor cortex include the superior colliculus (Fries, 1985), the mesopon-tine tegmentum (Hartmann-von Monakow et al., 1981), the thalamus (Kurata, 1994) and the striatum (Takada et al., 1998). The cuneus presents a whole set of hierarchical connections with other parastriate and extrastriate occipital areas (Zilles and Clarke, 1997) and is connected with the thalamus, notably the pulvinar (Adams et al., 2000). Areas of significant change within these sets of brain regions were determined using linear contrasts of the parameter estimates. The resulting set of voxel values for each contrast constituted a map of the t statistic [SPM $\{\mathrm{T}\}]$. Results were considered significant at small volume corrected $\mathrm{P}<0.05$, using a $10 \mathrm{~mm}$ radius-spherical volume of interest on our predetermined regions of interest.

\section{Acknowledgements}

This work was supported by the Fonds National de la Recherche Scientifique de Belgique (FNRS), the 
Fondation Medicale Reine Elisabeth, the Research Fund of ULg, and the PAI/IAP Interuniversity Pole of Attraction P4/22. S.L., A.C. and P.M. are respectively Postdoctoral Researcher, Research Associate and Senior Research Associate (FNRS). P.P. is supported by PAL Personal thanks are expressed to C. Mesters, G. and J. Hodiaumont, P. Hawotte. and J.L. Genon for technical assistance.

\section{References}

Adams, M.M., Hof, P.R., Gattass, R., Webster, M.J., Ungerleider, L.G., 2000. Visual cortical projections and chemoarchitecture of macaque monkey pulvinar. J. Comp. Neurol. 419, 377-393.

Amzica, F., Neckelmann, D., Steriade, M., 1997. Instrumental conditioning of fast (20- to 50-Hz) oscillations in corticothalamic networks. Proc. Natl. Acad. Sci. USA 94, 1985-1989.

Andersen, R.A., 1997. Multimodal integration for the representation of space in the posterior parietal cortex. Philos. Trans. R. Soc. Lond. B Biol. Sci. 352, 1421-1428.

Boecker, H., Dagher, A., Ceballos-Baumann, A.O., Passingham, R.E., Samuel, M., Friston, K.J., Poline, J., Dettmers, C, Conrad, B., Brooks. D.J., 1998. Role of the human rostral supplementary motor area and the basal ganglia in motor sequence control: investigations with H2 15 O PET. J. Neurophysiol. 79, 1070-1080.

Cleeremans, A., McClelland, J.L., 1991. Learning the structure of event sequences. J. Exp. Psychol. Gen. 120, $235-253$.

Fries, W., 1985. Inputs from motor and premotor cortex to the superior colliculus of the macaque monkey. Behav. Brain Res. 18, $95-105$.

Friston, K.J., 1997. Analysing brain images: principles and overview. In: Frackowiak, R.S.J., Friston, K.J., Frith, C.D., Dolan, R.J., Mazziotta. J.C. (Eds.), Analysing Brain Images: Principles and Overview. Academic Press, San Diego, CA, pp. $25-41$.

Friston, K.J., Buechel, C, Fink, G.R., Morris, J., Rolls, E., Dolan, R.J., 1997. Psychophysiological and modulatory interactions in neuroimaging. Neuroimage 6, 218-229.

Gais, S., Plihal, W, Wagner, U., Born, J., 2000. Early sleep triggers memory for early visual discrimination skills. Nat. Neurosci. $3,1335-1339$.

Hartmann-von Monakow, K., Akert, K., Kunzle, H., 1981. Projection of precentral, premotor and prefrontal cortex to the basilar pontine grey and to nucleus reticularis tegmenti pontis in the monkey (Macaca fascicularis). Schweiz Arch. Neurol. Neurochir. Psychiatry 129, 189-208.

Houk, J.C, Wise, S.P., 1995. Distributed modular architectures linking basal ganglia, cerebellum, and cerebral cortex: their role in planning and controlling action. Cereb. Cortex 5, 95-110.

Kurata, K., 1994. Site of origin of projections from the thalamus to dorsal versus ventral aspects of the premotor cortex of monkeys. Neurosci. Res. 21, 71-76.

Kurata, K, Tsuji, T., Naraki, S., Seino, M., Abe, Y., 2000. Activation of the dorsal premotor cortex and pre-supplementary motor area of humans during an auditory conditional motor task. J. Neurophysiol. 84, 1667-1672.

Lu, M.T., Preston, J.B., Strick, P.L., 1994. Interconnections between the prefrontal cortex and the premotor areas in the frontal lobe. J. Comp. Neurol. 341, 375-392.

Luppino, G, Matelli, M., Camarda, R., Rizzolatti, G, 1993. Corticocortical connections of area F3 (SMA-proper) and area F6 (pre-SMA) in the macaque monkey. J. Comp. Neurol. 338, 114-140.

Maquet, P., Laureys, S., Peigneux, P., Fuchs, S., Petiau, C, Phillips, C, Aerts, J., Del Fiore, G, Degueldre, C, Meulemans, T., Luxen, A. Franck, G, Van Der Linden, M, Smith, C, Cleeremans, A., 2000. Experience-dependent changes in cerebral activation during human REM sleep. Nat. Neurosci. 3, 831-836.

McGaugh, J.L., 2000. Memory - a century of consolidation. Science 287, 248-251.

Pandya, D.N., Vignolo, L.A., 1971. Intra- and interhemispheric projections of the precentral, premotor and arcuate areas in the rhesus monkey. Brain Res. 26, 217-233.

Peigneux, P., Maquet, P., Meulemans, T., Destrebecqz, A., Laureys, S., Degueldre, C, Delfiore, G, Aerts, J., Luxen, A., Franck, G, Van der Linden, M., Cleeremans, A., 2000. Striatum forever, despite sequence learning variability: a random effect analysis of PET data. Hum. Brain Mapp. 10, 179-194.

Pisella, L., Grea, H., Tilikete, C, Vighetto, A., Desmurget, M., Rode, G, Boisson, D., Rossetti, Y,, 2000. An 'automatic pilot' for the hand in human posterior parietal cortex: toward reinterpreting optic ataxia. Nat. Neurosci. 3, 729-736.

Rechtschaffen, A., Kales, A.A., 1968. A manual of standardized terminology, techniques and scoring system for sleep stages of human sujects. US Department of Health, Education and Welfare, Bethesda, 
Published in: Neuroscience (2001), vol. 105, iss. 3, pp. 521-525

Status: Postprint (Author's version)

MD. Rizzolatti, G, Arbib, M.A., 1998. Language within our grasp. Trends Neurosci. 21, 188-194.

Rizzolatti, G, Luppino, G, Matelli, M, 1998. The organization of the cortical motor system: new concepts. Electroencephalogr. Clin. Neurophysiol. 106, 283-296.

Sakai, K., Hikosaka, O., Miyauchi, S., Sasaki, Y., Fujimaki, N., Putz, B., 1999. Presupplementary motor area activation during sequence learning reflects visuo-motor association. J. Neurosci. 19, RC1.

Smith, C, 1995. Sleep states and memory processes. Behav. Brain Res. 69, 137-145.

Stickgold, R., James, L., Hobson, J.A., 2000. Visual discrimination learning requires sleep after training. Nat. Neurosci. 3, 12371238.525

Takada, M., Tokuno, H., Nambu, A., Inase, M., 1998. Corticostriatal projections from the somatic motor areas of the frontal cortex in the macaque monkey: segregation versus overlap of input zones from the primary motor cortex, the supplementary motor area, and the premotor cortex. Exp. Brain Res. 120, 114-128.

Talairach, J., Tournoux, P., 1988. Co-planar stereotaxic atlas of the human brain. Thieme-Verlag, Stuttgart.

Wilson, M.A., McNaughton, B.L., 1994. Reactivation of hippocampal ensemble memories during sleep. Science 265, 676-679.

Wise, S.P., Boussaoud, D., Johnson, P.B., Caminiti, R., 1997. Premotor and parietal cortex: corticocortical connectivity and combinatorial computations. Annu. Rev. Neurosci. 20, 25-42.

Wolpert, D.M., Goodbody, S.J., Husain, M., 1998. Maintaining internal representations: the role of the human superior parietal lobe. Nat. Neurosci. 1, 529-533.

Zilles, K., Clarke, S., 1997. In: Rockland, K.S., Kaas, J.H., Peters, A. (Eds.), Architecture, Connectivity, and Transmitter Receptors of Human Extrastriate Visual Cortex. Comparison with Non Human Primates, Vol. 12. Plenum, New York, pp. 673742 . 